\title{
Factors associated with time to independent walking recovery post-stroke
}

\author{
Caitlin Kennedy (D) ,' Julie Bernhardt (D) ,',3 Leonid Churilov (D) ,' \\ Janice M Collier (D), ${ }^{2}$ Fiona Ellery (D) , ${ }^{2}$ Venesha Rethnam (D) , \\ Lilian B Carvalho (D) ,"3 Geoffrey A Donnan (D) , 4,5 Kathryn S Hayward (D) 2,3,6
}

\begin{abstract}
Melbourne Medical School The University of Melbourne, Parkville, Victoria, Australia ${ }^{2}$ Florey Institute of Neuroscience and Mental Health, The University of Melbourne, Heidelberg, Victoria, Australia ${ }^{3}$ NHMRC Centre for Research Excellence in Stroke Rehabilitation and Brain Recovery, Melbourne, Victoria, Australia

${ }^{4}$ Melbourne Brain Centre, The Royal Melbourne Hospital, Parkville, Victoria, Australia ${ }^{5}$ The University of Melbourne, Parkville, Victoria, Australia ${ }^{6}$ Melbourne School of Health Sciences, The University of Melbourne, Melbourne, Victoria, Australia
\end{abstract}

\section{Correspondence to} Dr Kathryn S Hayward, Melbourne School of Health Sciences, The University of Melbourne, Melbourne, VIC 3010,Australia; kate.hayward@ unimelb.edu.au

Received 13 September 2020 Revised 19 January 2021 Accepted 20 January 2021 Published Online First 17 March 202

\section{ABSTRACT}

Background Past studies have inconsistently identified factors associated with independent walking post-stroke. We investigated the relationship between pre-stroke factors and factors collected acutely after stroke and number of days to walking $50 \mathrm{~m}$ unassisted using data from A Very Early Rehabilitation Trial (AVERT).

Methods The outcome was recovery of $50 \mathrm{~m}$ independent walking, tested from 24 hours to 3 months post-stroke. A set of a priori defined factors (participant demographics: age, sex, handedness; pre-stroke: hypertension, ischaemic heart disease, hypercholesterolaemia, diabetes mellitus, atrial fibrillation; stroke-related: stroke severity, stroke type, ischaemic stroke location, stroke hemisphere, thrombolysis) were investigated for association with independent walking using a cause-specific competing risk Cox proportional hazards model. Respective effect sizes are reported as cause-specific adjusted HR (caHR) adjusted for age, stroke severity and AVERT intervention. Results A total of 2100 participants (median age 73 years, National Institutes of Health Stroke Scale 7, <1\% missing data) with stroke were included. The median time to walking 50 m unassisted was 6 days (IQR 2-63) and $75 \%$ achieved independent walking by 3 months. Adjusted Cox regression indicated that slower return to independent walking was associated with older age (caHR 0.651, 95\% Cl 0.569 to 0.746), diabetes (caHR $0.836,95 \% \mathrm{Cl} 0.740$ to 0.945 ), severe stroke (caHR $0.094,95 \% \mathrm{Cl} 0.072$ to 0.122 ), haemorrhagic stroke (caHR $0.790,95 \% \mathrm{Cl} 0.675$ to 0.925 ) and right hemisphere stroke (caHR $0.796,95 \% \mathrm{Cl} 0.714$ to 0.887 ). Conclusion Our analysis provides robust evidence for important factors associated with independent walking recovery. These findings highlight the need for tailored mobilisation programmes that target subgroups, in particular people with haemorrhagic and severe stroke.

\section{INTRODUCTION}

Approximately two-thirds of people experience difficulty in walking independently after acute stroke, which persists for a large proportion of survivors at 3 months post-stroke. ${ }^{1}$ Stroke survivors have identified walking after stroke to be a high priority research area. ${ }^{2}$ Survivors are most interested in recovery of walking independence (ie, without hands-on help from another person ${ }^{3}$ ), compared with distance, appearance or speed. ${ }^{4}$ From a health service perspective, independent walking is important to inform decisions about rehabilitation goals and discharge destination. ${ }^{5}$ Therefore, delay to independent walking can significantly burden stroke survivors, their families and the healthcare system. ${ }^{67}$

Understanding the factors associated with recovery of independent walking is important to establish rehabilitation goals and inform discharge planning. A direct measure of walking (eg, 10 Metre Walk Test) is more informative of walking ability as compared with the modified Rankin Scale (mRS), which does not uniformly measure walking ability, ${ }^{8}$ yet has been used for this purpose in the past. In the context of a direct measure of walking, it appears that both pre-stroke and acute stroke factors may be associated with independent walking. Younger age is commonly positively associated with recovery of walking, ${ }^{9-13}$ while sex appears to have no association. ${ }^{9} 10$ 12-16 The evidence regarding stroke severity, ${ }^{9} 1415$ stroke type $\mathrm{e}^{101315}$ and presence of pre-stroke comorbidities, such as diabetes, ${ }^{9} 1213$ is inconclusive for the association with walking recovery. However, the evidence of association between these factors and direct walking outcomes is limited. ${ }^{17}$ There is little evidence for the association of pre-existing medical comorbidities with unassisted walking post-stroke. To date, the association of neuroimaging factors (eg, stroke type, stroke hemisphere, lesion volume) with independent walking has not been studied extensively. Therefore, using a direct walking measure in a large, representative sample ${ }^{18}$ that is relevant for community ambulation, such as number of days to walking $50 \mathrm{~m}$ unassisted, ${ }^{19}$ can help our understanding of pre-stroke and acute stroke factors that may be associated with independent walking.

In this study, we used data from A Very Early Rehabilitation Trial (AVERT), ${ }^{19}$ a large $(n=2104)$ international stroke rehabilitation trial. We aimed to investigate the association between a priori identified pre-stroke factors and factors collected acutely after stroke and number of days to walking $50 \mathrm{~m}$ unassisted.

\section{METHODS}

\section{Participants}

Participants enrolled in AVERT were included in this study. AVERT was a parallel-group, single-blind, international, multisite, randomised controlled trial conducted in 56 stroke units across five countries: Australia, New Zealand, Singapore, Malaysia and 
the UK (England, Northern Ireland, Scotland, Wales), between 18 July 2006 and 16 October 2014. Due to the exploratory aim of our study, all participants were treated as a single cohort and it is reported in accordance with Strengthening the Reporting of Observational Studies in Epidemiology guidelines. ${ }^{20}$

Participants were eligible for inclusion if aged 18 years or older, with a diagnosis of first or recurrent stroke (ischaemic or haemorrhagic stroke) and admitted to a stroke unit within 24 hours of stroke onset. Treatment with recombinant tissue plasminogen activator was permitted. Exclusion criteria were clinically significant pre-morbid disability $(\mathrm{mRS}>2)$, early deterioration, direct admission to an intensive care unit, palliative treatment, immediate surgery, serious medical illness, unstable coronary condition, subarachnoid haemorrhage, no response to voice, systolic blood pressure $<110 \mathrm{~mm} \mathrm{Hg}$ or $>220 \mathrm{~mm} \mathrm{Hg}$, oxygen saturation $<92 \%$ with oxygen supplementation, resting heart rate $<40 \mathrm{bpm}$ or $>110 \mathrm{bpm}$, temperature $>38.5^{\circ} \mathrm{C}$ or enrolment in a separate intervention trial. The AVERT intervention, consisting of a suite of early mobilisation and rehabilitation strategies, has been described elsewhere. ${ }^{19}$

\section{Days to walking $50 \mathrm{~m}$ unassisted}

The outcome of interest was number of days to walking $50 \mathrm{~m}$ unassisted post-stroke, which was measured as the difference between date of stroke (considered day 0 ) and date of first achieving walking over $50 \mathrm{~m}$ without the need for hands-on help of another person. ${ }^{3}$ The outcome was censored at 3 months as per the AVERT protocol, consistent with spontaneous neurological recovery primarily in the first 10 weeks post-stroke. ${ }^{21}$ Treating physiotherapists assessed participants for their ability to walk $50 \mathrm{~m}$ unassisted during acute hospitalisation. For participants unable to walk $50 \mathrm{~m}$ unassisted at the time of discharge, blinded assessors acquired this data using a variety of measures including contact with clinicians in rehabilitation facilities, review of medical records and detailed patient interview and assessment at 3 months.

\section{Variables of interest}

The independent variables of interest were categorised as participant demographics, pre-stroke and stroke-related factors. All independent variables were collected at baseline assessment, which took place within the first 24 hours from stroke onset unless not routinely feasible, for example, MRI. Participant demographics were age at stroke onset (years), sex (male, female) and handedness (left, right; hand used for writing). Prestroke variables were comorbidities noted in the medical history (hypertension, ischaemic heart disease, hypercholesterolaemia, diabetes mellitus, atrial fibrillation; all defined as present or absent). Stroke-related variables were stroke severity (National Institutes of Health Stroke Scale, NIHSS), ${ }^{22}$ considered as a continuous variable and in subgroups (mild (0-7), moderate $(8-16)$, severe $(>16))$, stroke type (ischaemic, haemorrhagic), Oxfordshire Stroke Classification ${ }^{23}$ subgroups (total anterior circulation infarct (TACI), partial anterior circulation infarct (PACI), posterior circulation infarct (POCI), lacunar infarct (LACI), intracerebral haemorrhage $(\mathrm{ICH})$ ), ischaemic stroke location (large cortical, small cortical, hemispheric lacunar, brainstem, cerebellum, other, no infarct on imaging), stroke hemisphere (left, right, brainstem, not evident on imaging, unknown) and thrombolysis treatment with recombinant tissue plasminogen activator (yes, no). Stroke-related variables of stroke type, ischaemic stroke location and stroke hemisphere were reported based on information from routine neuroimaging (CT or MRI) performed at the local hospital.

\section{Statistical analysis}

As reported elsewhere, ${ }^{19}$ a sample of 2104 participants in AVERT was estimated to provide $80 \%$ power to detect a significant intervention effect. The statistical analysis plan for AVERT was pre-specified and published elsewhere. ${ }^{3}$ For the purpose of the current study, the intervention and control groups were combined into a single cohort due to no meaningful effect of early mobilisation on the number of days to walking $50 \mathrm{~m}$ unassisted. $^{19}$

The number of days from stroke onset to walking $50 \mathrm{~m}$ unassisted was used as the time variable for participants who walked, including those who walked and subsequently died within the 3 -month period. The number of days from stroke onset to death was used as the time variable for participants who died and did not walk within the 3 -month period. The censoring time of 3 months (range of 89-92 days depending on month of recruitment) was allocated to participants who did not walk and did not die within 3 months. Participants with missing data at 3 months were excluded from statistical analysis. ${ }^{24}$ Since the event of death provides informative censoring for walking, that is, participants who died are clearly not expected to achieve walking after the event of death, death is considered as a competing risk to the event of walking.

Under the circumstances of informative censoring, the use of standard Cox proportional hazards regression is not appropriate and cause-specific modelling was undertaken. A cause-specific competing risk Cox proportional hazards model ${ }^{25}$ for days to unassisted walking was used, with death as the competing risk. Competing risks are present when a participant is at risk of more than one mutually exclusive event and the occurrence of one of these will prevent another other event from occurring. In our model, the event of death can prevent a participant from walking. In order to investigate the association between the variables of interest and the outcome (days to walking $50 \mathrm{~m}$ unassisted), the dependent variable was censored at 3 months for all models. All models were adjusted for a set of prognostic covariates prespecified in the statistical analysis plan for the AVERT trial ${ }^{3}$ : age, stroke severity (NIHSS) and AVERT intervention group. Models for Oxfordshire Stroke Classification and ischaemic stroke location were not adjusted for stroke severity due to excessive collinearity. Individual effect sizes were estimated as a causespecific adjusted HR (caHR) with corresponding 95\% CI. No single multivariable model that included all individual factors was presented due to potential complex confounding where there is an overlap in variability in outcome explained by different factors. ${ }^{26}$ For a priori selected prognostic factors, we present the outcome for both walking and death to emphasise the informative censoring nature of death relative to walking. All statistical analysis was performed using STATA/IC, V.15.1 (StataCorp).

\section{RESULTS}

\section{Demographics}

From 2104 participants in AVERT, the median age at stroke onset was 73 years (IQR 63-80). There were 1286 (61\%) male participants. The median NIHSS Score was 7 (IQR 4-12). There were 1846 (88\%) participants with ischaemic stroke. Participant characteristics are summarised in table 1.

\section{Days to walking 50 m unassisted}

Data for number of days to walking $50 \mathrm{~m}$ unassisted by 3 months was available for $2100(99.8 \%)$ participants. Four $(0.2 \%)$ 
Table 1 Independent variables, walking status by 3 months post-stroke and median number of days to walking $50 \mathrm{~m}$ unassisted

\begin{tabular}{|c|c|c|c|c|}
\hline \multirow[b]{2}{*}{ Independent variables } & \multirow{2}{*}{$\begin{array}{l}\text { All participants } \\
(n=2104) \\
n(\%)\end{array}$} & \multicolumn{2}{|c|}{$\begin{array}{l}\text { Achieved } 50 \mathrm{~m} \text { unassisted walk by } 3 \text { months } \\
\text { post-stroke }\left(\mathrm{n}=2100^{*}\right)\end{array}$} & \multirow{2}{*}{$\begin{array}{l}\text { Median days to } 50 \mathrm{~m} \text { unassisted walk } \\
\left(\mathrm{n}=2100^{*}\right)\end{array}$} \\
\hline & & $\begin{array}{l}\text { No, } \\
\mathrm{n}(\%)\end{array}$ & $\begin{array}{l}\text { Yes, } \\
\text { n (\%) }\end{array}$ & \\
\hline \multicolumn{5}{|l|}{ Participant demographics } \\
\hline Participants & $2104(100)$ & $520(25)$ & $1580(75)$ & $6(2-63)$ \\
\hline Age, years (median (IQR)) & $72.5(62.9-80.3)$ & $77.3(68.0-82.7)$ & $71.0(61.0-79.2)$ & N/A \\
\hline$<65$ & $629(30)$ & $94(18)$ & $532(34)$ & $4(2-29)$ \\
\hline $65-80$ & $929(44)$ & $230(44)$ & $698(44)$ & $7(2-70)$ \\
\hline$>80$ & $546(26)$ & $196(38)$ & $350(22)$ & 11 ( 3 to $>90 d)$ \\
\hline \multicolumn{5}{|l|}{ Sex } \\
\hline Female & $818(39)$ & $223(43)$ & $594(38)$ & $8(2-82)$ \\
\hline Male & $1286(61)$ & $297(57)$ & $986(62)$ & $5(2-51)$ \\
\hline \multicolumn{5}{|l|}{ Handedness } \\
\hline Left & $135(6)$ & $22(4)$ & $113(7)$ & $5(2-35)$ \\
\hline Right & $1960(93)$ & $492(95)$ & 1465 (93) & $6(2-66)$ \\
\hline \multicolumn{5}{|l|}{ Pre-stroke factors } \\
\hline Hypertension & $1424(68)$ & $370(71)$ & $1051(67)$ & $7(2-76)$ \\
\hline Ischaemic heart disease & $486(23)$ & $145(28)$ & $341(22)$ & $8(2$ to $>90 d)$ \\
\hline Hypercholesterolaemia & $842(40)$ & $216(42)$ & $624(39)$ & $6(2-69)$ \\
\hline Diabetes mellitus & $467(22)$ & $139(27)$ & $326(21)$ & $9(2$ to $>90 d)$ \\
\hline Atrial fibrillation & $465(22)$ & $167(32)$ & $298(19)$ & $8(2$ to $>90 d)$ \\
\hline \multicolumn{5}{|l|}{ Stroke-related factors } \\
\hline \multicolumn{5}{|l|}{ Stroke severity } \\
\hline NIHSS Score, median (IQR) & $7(4-12)$ & $15(10-20)$ & $6(3-8)$ & $\mathrm{N} / \mathrm{A}$ \\
\hline Mild (1-7) & $1170(56)$ & $78(15)$ & $1088(69)$ & $3(1-7)$ \\
\hline Moderate (8-16) & $643(30)$ & $210(40)$ & $433(27)$ & $24(4$ to $>90 d)$ \\
\hline Severe $(>16)$ & $291(14)$ & $232(45)$ & $59(4)$ & $>90 \mathrm{~d}(85$ to $>90 \mathrm{~d})$ \\
\hline \multicolumn{5}{|l|}{ Stroke type } \\
\hline Ischaemic & $1846(88)$ & $440(85)$ & $1402(89)$ & $5(2-57)$ \\
\hline Haemorrhagic & $258(12)$ & $80(15)$ & $178(11)$ & 26 ( 5 to $>90 d)$ \\
\hline \multicolumn{5}{|l|}{ Oxfordshire Stroke Classification } \\
\hline $\mathrm{LACl}$ & $523(25)$ & $63(12)$ & $458(29)$ & $3(2-22)$ \\
\hline $\mathrm{TACl}$ & $456(22)$ & $260(50)$ & $196(12)$ & $>90 \mathrm{~d}(8$ to $>90 \mathrm{~d})$ \\
\hline $\mathrm{PACl}$ & $668(32)$ & $94(18)$ & $573(36)$ & $4(2-16)$ \\
\hline $\mathrm{POCl}$ & $199(9)$ & $23(4)$ & $175(11)$ & $3(2-10)$ \\
\hline $\mathrm{ICH}$ & $258(12)$ & $80(15)$ & $178(11)$ & $26(5$ to $>90 d)$ \\
\hline \multicolumn{5}{|c|}{ Ischaemic stroke location $(n=1845 t)$} \\
\hline Hemispheric lacunar & $209(11)$ & $27(6)$ & $182(13)$ & $4(2-18)$ \\
\hline Large cortical & $545(30)$ & $245(56)$ & $299(21)$ & 37 (4 to $>90 d$ ) \\
\hline Small cortical & $225(12)$ & $32(7)$ & $193(14)$ & $3(2-13)$ \\
\hline Brainstem & $56(3)$ & $8(2)$ & $48(3)$ & $4(2-42)$ \\
\hline Cerebellum & $57(3)$ & $9(2)$ & $48(3)$ & $5(2-26)$ \\
\hline Other & $102(6)$ & $26(6)$ & $76(5)$ & $9(2-79)$ \\
\hline No infarct on imaging & $651(35)$ & $93(21)$ & $555(40)$ & $3(2-13)$ \\
\hline \multicolumn{5}{|l|}{ Stroke hemisphere } \\
\hline Left & 819 (39) & $218(42)$ & $599(38)$ & $7(2-73)$ \\
\hline Right & $997(47)$ & $263(51)$ & $732(46)$ & $8(2-80)$ \\
\hline Brainstem & $41(2)$ & $8(2)$ & $33(2)$ & $4(2-42)$ \\
\hline Not evident on imaging & $239(11)$ & $27(5)$ & $212(13)$ & $3(1-7)$ \\
\hline Unknown & $8(<1)$ & $4(1)$ & $4(<1)$ & N/A \\
\hline Thrombolysis & 507 (24) & $147(28)$ & $360(23)$ & $6(2$ to $>90 d)$ \\
\hline
\end{tabular}

Data are $\mathrm{n}(\%)$ or median (IQR).

$>90 \mathrm{~d}$ Days to walking $50 \mathrm{~m}$ unassisted was outside the three month censoring period for the median or $75 \%$ percentile; this parameter was therefore not able to be defined by the analysis. Three months ranged from 89 to 92 days depending on month of recruitment.

N/A Median days to walking $50 \mathrm{~m}$ unassisted unable to be calculated by the statistical analysis.

${ }^{*}$ Missing data for walking status at 3 months and number of days to walking $50 \mathrm{~m}$ unassisted for four participants.

tMissing data for one participant; did not consent for neuroimaging.

ICH, intracerebral haemorrhage; LACI, lacunar infarct; NIHSS, National Institutes of Health Stroke Scale; PACl, partial anterior circulation infarct; POCI, posterior circulation infarct;

$\mathrm{TACl}$, total anterior circulation infarct. 
A

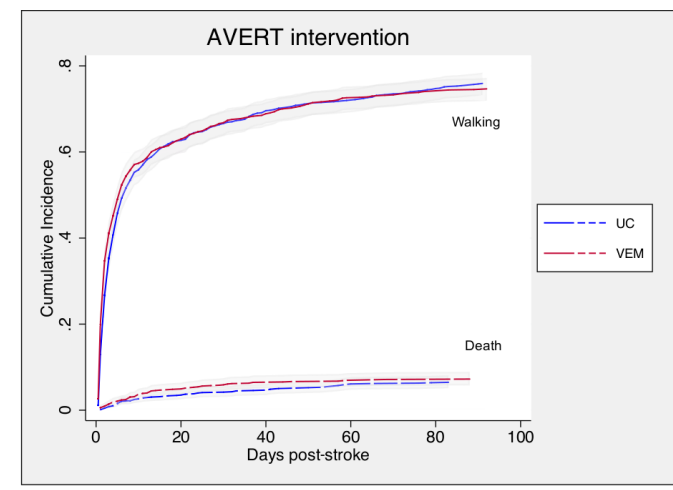

B

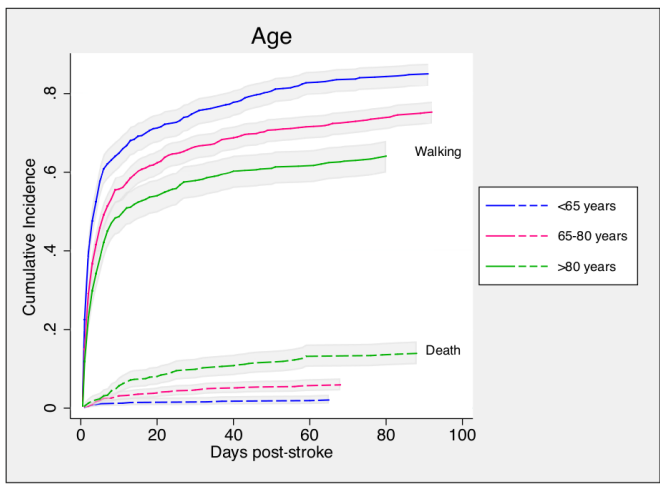

C

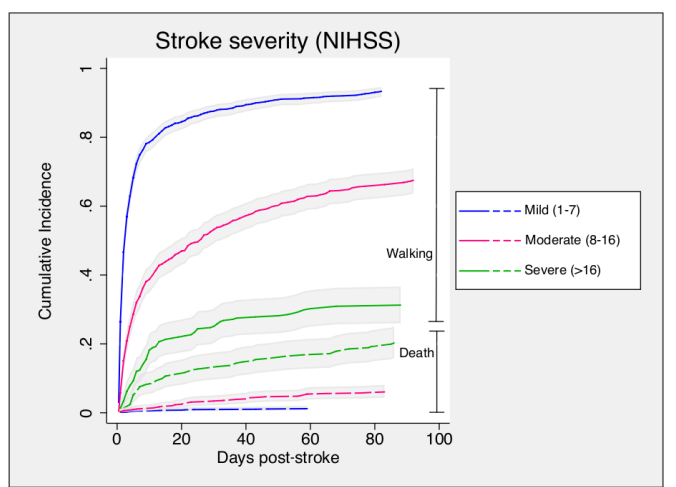

Figure 1 Association of avert intervention (A), age (B) and stroke severity (C) with number of days to walking $50 \mathrm{~m}$ unassisted or death by 3 months post-stroke. Figures are not adjusted for age, stroke severity or AVERT intervention. Shaded areas represent corresponding 95\% CI. AVERT, A Very Early Rehabilitation Trial; NIHSS, National Institutes of Health Stroke Scale; UC, usual care; VEM, very early mobilisation.

participants had missing data. Of these, three participants were alive, however, data were unavailable, and one participant could not be located (unknown if alive or dead). By 3 months after stroke, $1580(75 \%)$ participants had walked $50 \mathrm{~m}$ unassisted. Median number of days to walking $50 \mathrm{~m}$ unassisted was 6 days (IQR 2-63). Walking status by 3 months post-stroke (walking outcome achieved vs not achieved) and median number of days to walking for each participant subgroup are summarised in table 1.

\section{Factors associated with days to walking 50 m unassisted}

The relationship between factors of AVERT intervention, age and stroke severity with the number of days to walking $50 \mathrm{~m}$ unassisted are represented in figure 1, without adjustment for covariates.

Delayed return to unassisted walking post-stroke was independently associated with older age (caHR 0.651, 95\% CI 0.569 to 0.746 ), diabetes (caHR $0.836,95 \%$ CI 0.740 to 0.945 ), severe stroke (caHR $0.094,95 \% \mathrm{CI} 0.072$ to 0.122 ), haemorrhagic stroke (caHR $0.790,95 \% \mathrm{CI} 0.675$ to 0.925 ), right hemisphere stroke (caHR $0.796,95 \% \mathrm{CI} 0.714$ to 0.887 ), large cortical ischaemic stroke (caHR $0.439,95 \% \mathrm{CI} 0.397$ to 0.480 ), brainstem ischaemic stroke (caHR $0.779,95 \% \mathrm{CI} 0.653$ to 0.906 ) and Oxfordshire Stroke Classification categories of total anterior circulation infarct (TACI) (caHR $0.314,95 \%$ CI 0.287 to 0.341 ) and intracerebral haemorrhage (ICH) (caHR 0.527, 95\% CI 0.480 to 0.574 ) (figure 2). Earlier return to walking post-stroke was associated with small cortical ischaemic stroke (caHR 1.127, 95\% CI 1.010 to 1.244 ) and Oxfordshire Stroke Classification category of posterior circulation infarct (POCI) (caHR 1.124, 95\% CI 1.024 to 1.225 ) (figure 2).

Similar patterns of walking recovery post-stroke were found in patient subgroups for characteristics of sex, handedness, hypertension, ischaemic heart disease, hypercholesterolaemia, atrial fibrillation and thrombolysis (figure 2).

\section{DISCUSSION}

In this large, international cohort of acute stroke patients, we demonstrated that several pre-stroke and acute stroke factors are associated with recovery of walking $50 \mathrm{~m}$ unassisted at 3 months post-stroke. Baseline level of stroke severity had the most profound association with recovery of walking. Participants with severe and, to a lesser extent, moderate stroke were less likely to walk independently than participants with mild stroke. Initial stroke severity is highly predictive of long-term patient outcomes, such as performance in activities of daily living, ${ }^{17}$ as well as level of disability and dependence post-stroke. ${ }^{27}$ However, our findings have additionally demonstrated the importance of initial stroke severity for independent walking, a highly valued outcome for survivors post-stroke, ${ }^{4}$ but one which is inadequately captured by common post-stroke outcomes (eg, $\mathrm{mRS}) .^{8}$ The observed significant differences between the levels of severity (ie, mild, moderate, severe) and long-term walking outcomes signifies the need to investigate appropriate tailoring of rehabilitation interventions to each severity subgroup in future clinical trials.

Our findings demonstrated slower recovery of unassisted walking in participants with haemorrhagic stroke as compared with ischaemic stroke. The few studies that have investigated walking recovery in patients with ischaemic and haemorrhagic stroke found no difference between groups, however, sample size was fewer than 75 participants. ${ }^{10} 1315$ Individuals with haemorrhagic stroke may require a longer admission to rehabilitation compared with individuals with ischaemic stroke due to a slower recovery trajectory. Interestingly, by 3 months poststroke, the chance of walking was similar for participants with haemorrhagic and ischaemic strokes. This finding may be due to initial haematoma expansion ${ }^{28}$ as well as increasing cerebral oedema, which usually occurs within 2 days but can persist up to 3 weeks post-stroke. ${ }^{29}$ Both of these factors may contribute to clinicians and rehabilitation therapists being more cautious to initiate walking rehabilitation. The slower recovery seen in patients with haemorrhagic stroke in this study gives further weight to current efforts to find more effective treatments for this group of patients.

The variation in time taken to recover independent walking between individuals with ischaemic and haemorrhagic stroke has different implications on the healthcare system. The slower recovery trajectory seen with individuals with haemorrhagic stroke suggests that decisions about discharge destination, 
Cerebrovascular disease

Independent variables

Participant demographics

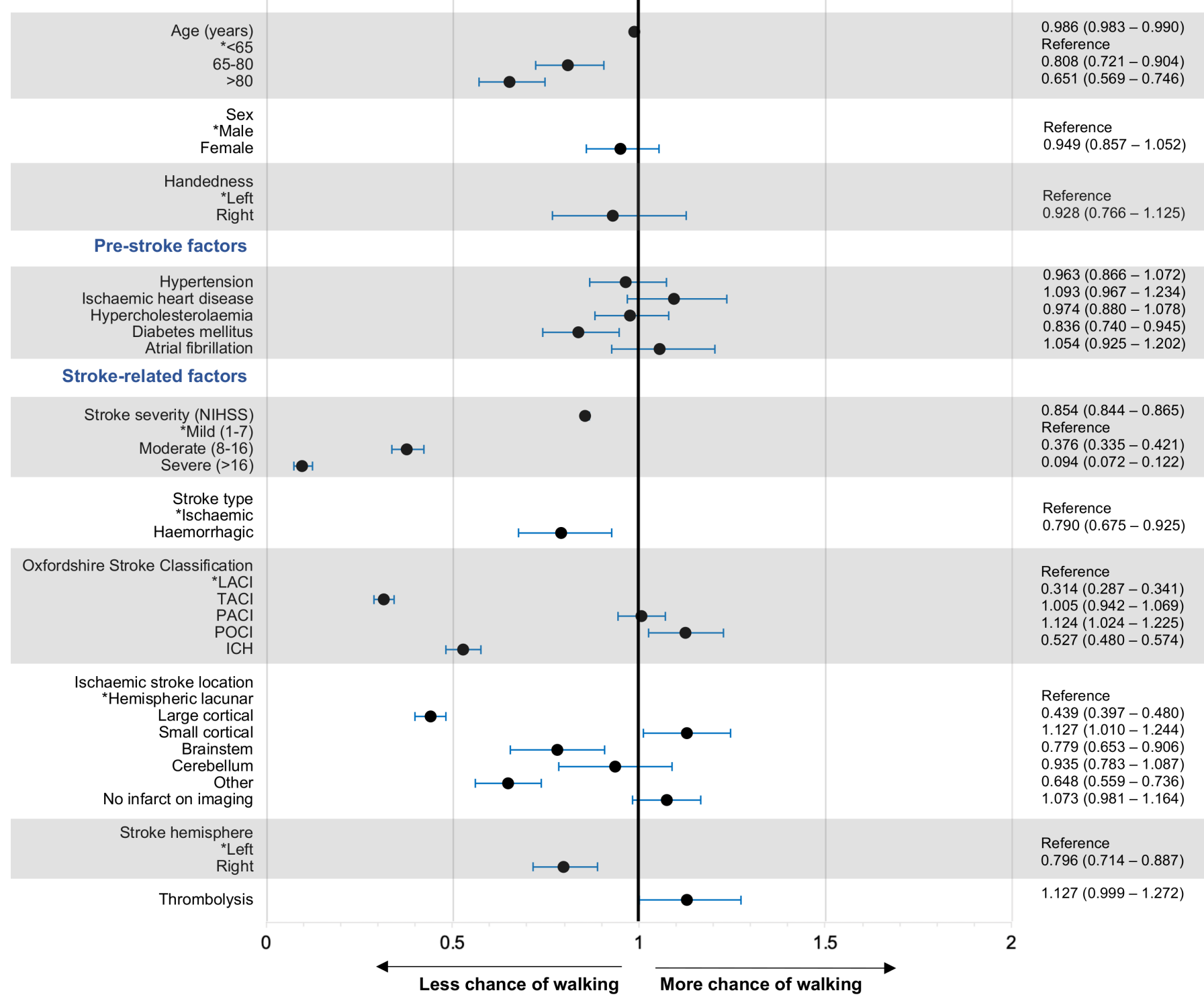

Figure 2 Cause-specific adjusted HRs and $95 \%$ Cl for association of independent variables and number of days to walking $50 \mathrm{~m}$ unassisted. Analyses are adjusted for age, stroke severity and AVERT intervention. Oxfordshire Stroke Classification and ischaemic stroke location are not adjusted for stroke severity due to excessive collinearity. ${ }^{*}$ Denotes reference group for each independent variable. caHR, cause-specific adjusted hazard ratio; ICH, intracerebral haemorrhage; LACI, lacunar infarct; NIHSS, National Institutes of Health Stroke Scale; PACl, partial anterior circulation infarct; POCl, posterior circulation infarct; TACl, total anterior circulation infarct.

particularly to residential care facilities, should be delayed. In contrast, the earlier return to walking in patients with ischaemic stroke may result in therapists not prioritising ongoing walking rehabilitation, despite their likely ongoing need, which may only be evident in the long-term. This difference in recovery profile suggests that tailored rehabilitation based on stroke type could be beneficial for optimising walking independence.

Unsurprisingly, our findings complement previous research which has demonstrated that younger individuals are more likely to return to independent walking post-stroke. ${ }^{9-13}$ In future stroke recovery trials, consideration should be given to age as an important stratification variable as well as accounting for it in statistical analysis. Interestingly, our findings demonstrated that diabetes is associated with delayed walking recovery after stroke. This finding may be attributed to the impact of chronic diabetic complications, including peripheral neuropathy, ${ }^{30}$ on walking and should be considered as an important pre-stroke comorbidity. However, past findings regarding the relationship between diabetes and post-stroke disability and functional outcomes have been mixed. ${ }^{913} 31-33$

While limited evidence was available regarding hemisphere of stroke, we found that right hemisphere stroke survivors have delayed recovery of walking as compared with left hemisphere stroke survivors. This finding may be attributed to greater presentation of perceptual disturbances and neglect in right hemisphere stroke. ${ }^{34} 35$ Spatial disturbance may impair confidence in mobilising without hands-on assistance, therefore delaying independent walking recovery.

Our study had a number of strengths. Data were drawn from a large, international trial with low loss to follow-up $(<1 \%)$. This 
trial recruited participants suitable for mobility rehabilitation and is generally comparable to population-based norms with a representative proportion of moderate to severe ischaemic and haemorrhagic stroke. ${ }^{18}$ Our outcome of interest, days to walking $50 \mathrm{~m}$ unassisted, is a reproducible and valid measure. ${ }^{36}$ Previous studies have often utilised shorter or timed distance measures (ie, 10 Metre Walk Test). ${ }^{11} 123738$ However, a longer distance outcome (ie, $50 \mathrm{~m}$ ) is important to reflect functional ambulation within the community setting. Finally, we used a robust causespecific competing risk model that appropriately accounts for competing risks.

Our study had some limitations. First, it is an analysis of data collected during AVERT, which focused on a primary disability outcome. As such, certain variables of interest identified from literature on walking rehabilitation were not collected, including pre-stroke fitness or activity level, body mass index and level of social support. Pre-stroke activity level and cognitive reserve are now considered important areas for ongoing research. ${ }^{39}$ Future clinical trials and inception cohort studies should consider collecting these additional pre-stroke factors. Second, we censored the outcome of interest at 3 months post-stroke. This endpoint was chosen to maintain consistency with the primary outcome reported in AVERT, mRS at 3 months. ${ }^{19}$ While the majority of stroke survivors recover the ability to walk by 3 months after stroke, ${ }^{1}$ some participants may have achieved this milestone after this period. Based on the findings from this study, future investigation of walking with censoring at 6 or 12 months post-stroke could be considered, particularly for research that includes individuals with haemorrhagic stroke and severe stroke. Finally, the method to index achievement of the outcome of interest (days to walking $50 \mathrm{~m}$ unassisted) did differ between participants, for example, reported by clinicians in rehabilitation facilities, review of medical records or detailed patient interview and assessment. However, the determination was always completed by a trained blinded assessor, which provided a consistent approach to classification of the outcome.

\section{CONCLUSION}

We found that older patients and those with diabetes, severe stroke, haemorrhagic stroke and right hemisphere stroke have a delayed return to independent walking. Our findings suggest the need for tailored stroke rehabilitation programmes that target meaningful subgroups, in particular people with haemorrhagic and severe stroke. This study also highlights the importance of stratifying participants in clinical trials based on age and stroke severity, or to control for these confounding factors in statistical analysis. These findings will be useful to guide future clinical trials in stroke recovery and contextualise clinical practice guideline recommendations.

\section{Twitter Kathryn S Hayward @kate_hayward_}

Acknowledgements The authors acknowledge the AVERT Trial Collaboration group investigators for allowing access to use the AVERT dataset to conduct this study. The authors thank all participants, families and assessors involved in the trial. The Florey Institute of Neuroscience and Mental Health acknowledges the support of the Victorian Government's Operational Infrastructure Support Grant.

Contributors JB, LC, JMC, FE and GAD contributed to major role in the acquisition of data. CK, LC and KSH designed and conceptualised study. CK, LC and KSH analysed and interpreted the data. JB interpreted the data. CK, LC and KSH drafted the manuscript for intellectual content. JB, JMC, FE, LBC, VR and GAD revised the manuscript for intellectual content.

Funding AVERT was funded by National Health and Medical Research Council (NHMRC) of Australia (386201, 1041401); Chest Heart and Stroke Scotland (Res08/ A114); Northern Ireland Chest Heart and Stroke (AVERT-NI 2008); Singapore Health (SHF/FG401P/2008); The Stroke Association, UK (TSA2009/09) and National Institute of Health Research, UK (HTA Project 12/01/16). NHMRC fellowship funding was provided to $\mathrm{KH}(1088449)$ and JB $(1058635,1154904)$. JB also received fellowship funding from Australian Research Council (0991086) and the National Heart Foundation.

Competing interests None declared.

\section{Patient consent for publication Not required.}

Ethics approval Ethics approval for AVERT was obtained from institutional review boards at all participating sites. Additional ethics approval for this analysis was received from Austin Health Human Research Ethics Committee (HREC), reference number LNR/19/Austin/15. AVERT was registered with the Australian New Zealand Clinical Trials Registry (ACTRN12606000185561), US National Institutes of Health (ISRCTN98129255) and UK Clinical Trials Gateway (ISRCTN98129255).

Provenance and peer review Not commissioned; externally peer reviewed.

Data availability statement Data are available upon reasonable request to AVERT Executive Committee.

\section{ORCID iDs}

Caitlin Kennedy http://orcid.org/0000-0002-3344-4674 Julie Bernhardt http://orcid.org/0000-0002-2787-8484 Leonid Churilov http://orcid.org/0000-0002-9807-6606 Janice M Collier http://orcid.org/0000-0003-2950-4870

Fiona Ellery http://orcid.org/0000-0002-7302-1895

Venesha Rethnam http://orcid.org/0000-0002-0493-8536

Lilian B Carvalho http://orcid.org/0000-0002-9803-7631

Geoffrey A Donnan http://orcid.org/0000-0001-6324-3403

Kathryn S Hayward http://orcid.org/0000-0001-5240-3264

\section{REFERENCES}

1 Jørgensen $\mathrm{HS}$, Nakayama $\mathrm{H}$, Raaschou $\mathrm{HO}$, et al. Recovery of walking function in stroke patients: the Copenhagen stroke study. Arch Phys Med Rehabil 1995:76:27-32.

2 Rudberg A-S, Berge E, Laska A-C, et al. Stroke survivors' priorities for research related to life after stroke. Top Stroke Rehabil 2020:1-6.

3 Bernhardt J, Churilov L, Dewey H, et al. Statistical analysis plan (SAP) for A Very Early Rehabilitation Trial (AVERT): an international trial to determine the efficacy and safety of commencing out of bed standing and walking training (very early mobilization) within $24 \mathrm{~h}$ of stroke onset vs. usual stroke unit care. Int J Stroke 2015;10:23-4.

4 Bohannon RW, Horton MG, Wikholm JB. Importance of four variables of walking to patients with stroke. Int J Rehabil Res 1991;14:246-50.

5 Frank M, Conzelmann M, Engelter S. Prediction of discharge destination after neurological rehabilitation in stroke patients. Eur Neurol 2010;63:227-33.

6 Australian Institute of Health and Welfare. Australia's health 2018: in brief. Cat. no. AUS 222, 2018. Available: https://www.aihw.gov.au/getmedia/fe037cf1-0cd0-4663a8c0-67cd09b1f30c/aihw-aus-222.pdf.aspx?inline=true [Accessed 20 Jul 2020].

7 Deloitte Access Economics. The economic impact of stroke in Australia, 2013. Available: https://www2.deloitte.com/au/en/pages/economics/articles/economicimpact-stroke-australia.html [Accessed 20 Jul 2020].

8 Rethnam V, Bernhardt J, Johns H, et al. Look closer: the multidimensional patterns of post-stroke burden behind the modified Rankin scale. Int I Stroke 2020:174749302095194 (Published online first: 27 August 2020).

9 Cumming TB, Thrift AG, Collier JM, et al. Very early mobilization after stroke fast-tracks return to walking: further results from the phase II avert randomized controlled trial. Stroke 2011;42:153-8.

10 Hirano $Y$, Hayashi T, Nitta 0 , et al. Prediction of independent walking ability for severely hemiplegic stroke patients at discharge from a rehabilitation Hospital. J Stroke Cerebrovasc Dis 2016:25:1878-81.

11 Mahendran N, Kuys SS, Brauer SG. Which impairments, activity limitations and personal factors at hospital discharge predict walking activity across the first 6 months poststroke? Disabil Rehabil 2020;42:763-9.

12 Mayo NE, Korner-Bitensky NA, Becker R. Recovery time of independent function poststroke. Am J Phys Med Rehabil 1991;70:5-12.

13 Wang $Y-H$, Yang Y-R, Pan P-J, et al. Modeling factors predictive of functional improvement following acute stroke. J Chin Med Assoc 2014;77:469-76.

14 Aaslund MK, Moe-Nilssen R, Gjelsvik BB, et al. A longitudinal study investigating how stroke severity, disability, and physical function the first week post-stroke are associated with walking speed six months post-stroke. Physiother Theory Pract 2017:33:932-42.

15 Smith M-C, Barber PA, Stinear CM. The twist algorithm predicts time to walking independently after stroke. Neurorehabil Neural Repair 2017;31:955-64.

16 Veerbeek JM, Van Wegen EEH, Harmeling-Van der Wel BC, et al. Is accurate prediction of gait in nonambulatory stroke patients possible within 72 hours poststroke? the EPOS study. Neurorehabil Neural Repair 2011;25:268-74.

17 Kwakkel G, Veerbeek JM, van Wegen EEH, et al. Predictive value of the NIHSS for ADL outcome after ischemic hemispheric stroke: does timing of early assessment matter? J Neurol Sci 2010;294:57-61. 
18 Bernhardt J, Raffelt A, Churilov L, et al. Exploring threats to generalisability in a large international rehabilitation trial (AVERT). BMJ Open 2015;5:e008378.

19 AVERT Trial Collaboration group. Efficacy and safety of very early mobilisation within $24 \mathrm{H}$ of stroke onset (AVERT): a randomised controlled trial. Lancet 2015;386:46-55.

20 Vandenbroucke JP, von Elm E, Altman DG, et al. Strengthening the reporting of observational studies in epidemiology (STROBE): explanation and elaboration. PLoS Med 2007;4:e297.

21 Kwakkel G, Kollen BJ. Predicting activities after stroke: what is clinically relevant? Int $J$ Stroke 2013;8:25-32.

22 Kasner SE, Chalela JA, Luciano JM, et al. Reliability and validity of estimating the NIH stroke scale score from medical records. Stroke 1999:30:1534-7.

23 Wardlaw JM, Dennis MS, Lindley RI, et al. The validity of a simple clinical classification of acute ischaemic stroke. J Neurol 1996:243:274-9.

24 Kang $\mathrm{H}$. The prevention and handling of the missing data. Korean J Anesthesiol 2013;64:402-6.

25 Noordzij M, Leffondré K, van Stralen KJ, et al. When do we need competing risks methods for survival analysis in nephrology? Nephrol Dial Transplant 2013;28:2670-7.

26 Westreich D, Greenland S. The table 2 fallacy: presenting and interpreting confounder and modifier coefficients. Am J Epidemiol 2013;177:292-8.

27 Sucharew H, Khoury J, Moomaw CJ, et al. Profiles of the National Institutes of health stroke scale items as a predictor of patient outcome. Stroke 2013;44:2182-7.

28 Kazui S, Naritomi H, Yamamoto H, et al. Enlargement of spontaneous intracerebral hemorrhage. incidence and time course. Stroke 1996:27:1783-7.

29 Zazulia AR, Diringer MN, Derdeyn CP, et al. Progression of mass effect after intracerebral hemorrhage. Stroke 1999:30:1167-73.
30 Menz HB, Lord SR, St George R, et al. Walking stability and sensorimotor function in older people with diabetic peripheral neuropathy. Arch Phys Med Rehabil 2004;85:245-52

31 Karapanayiotides T, Piechowski-Jozwiak B, van Melle G, et al. Stroke patterns, etiology, and prognosis in patients with diabetes mellitus. Neurology 2004;62:1558-62.

32 Ripley DL, Seel RT, Macciocchi SN, et al. The impact of diabetes mellitus on stroke acute rehabilitation outcomes. Am J Phys Med Rehabil 2007;86:754-61.

33 Nannetti L, Paci M, Baccini M, et al. Recovery from stroke in patients with diabetes mellitus. J Diabetes Complications 2009;23:249-54.

34 Vallar G, Perani D. The anatomy of unilateral neglect after right-hemisphere stroke lesions. A clinical/CT-scan correlation study in man. Neuropsychologia 1986;24:609-22.

35 Chen P, Chen CC, Hreha K, et al. Kessler Foundation neglect assessment process uniquely measures spatial neglect during activities of daily living. Arch Phys Med Rehabil 2015:96:869-76.

36 Hachiya M, Murata S, Otao H, et al. Reproducibility and validity of the 50-meter walking test in community-dwelling elderly. J Phys Ther Sci 2015;27:1511-4.

37 Strømmen AM, Christensen T, Jensen K. Intensive treadmill training in the acute phase after ischemic stroke. Int J Rehabil Res 2016:39:145-52.

38 Yelnik AP, Quintaine V, Andriantsifanetra C, et al. AMOBES (active mobility very early after stroke): a randomized controlled trial. Stroke 2017:48:400-5.

39 Kwakkel G, Lannin NA, Borschmann K, et al. Standardized measurement of sensorimotor recovery in stroke trials: consensus-based core recommendations from the stroke recovery and rehabilitation roundtable. Int I Stroke 2017:12:451-61. 